\title{
Germinación de semillas de anón (Annona squamosa L.) sometidas a estratificación
}

\section{Germination of sugar apple (Annona squamosa L.) seeds submitted to estratification}

NATALIA ELIZABETH MORENO B..$^{1,4}$

DIEGO MIRANDA ${ }^{2}$

FABIO ERNESTO MARTÍNEZ M. ${ }^{3}$

Frutos de anón del proyecto Banco de Germoplasma de Anón.

Foto: S.L. Castañeda y D. Miranda

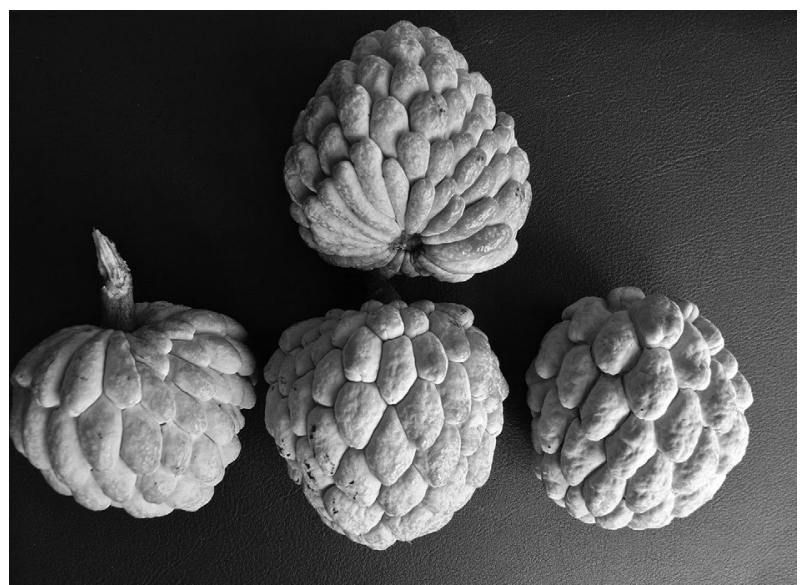

\section{RESUMEN}

En esta investigación se estudió el efecto de la estratificación sobre la germinación de semillas de anón, provenientes de cultivos ubicados en Apulo, Cundinamarca. Se evaluó el efecto de la estratificación húmeda fría $\left(8^{\circ} \mathrm{C}\right)(\mathrm{EHF})$ y la estratificación húmeda fría seguida de estratificación húmeda caliente $\left(25^{\circ} \mathrm{C}\right)(\mathrm{EHF}+\mathrm{EFC})$ durante 15, 30 y 45 días para cada tratamiento. Posterior a la aplicación de estos tratamientos se realizaron pruebas de germinación en fitotrones a 30 y $35^{\circ} \mathrm{C}$ para determinar el efecto de la temperatura de estratificación sobre la germinación. Las semillas presentaron mayores porcentajes de germinación (PG) y velocidad media de germinación (VMG) con la aplicación del tratamiento de EHF durante 45 días a $35^{\circ} \mathrm{C}$ con PG de $65,6 \%$. La germinación a $30^{\circ} \mathrm{C}$ tardó más y mostró PG y VMG más bajos que la mayoría de los tratamientos a $35^{\circ} \mathrm{C}$. A partir de los resultados se determinó que las semillas de anón mejoran su germinabilidad a temperaturas de $35^{\circ} \mathrm{C}$; adicionalmente, la respuesta de las semillas a los tratamientos de estratificación húmeda en frío y calor (EHF y EHF+EHC), podría indicar la presencia de latencia morfofisiológica en este tipo de semillas.

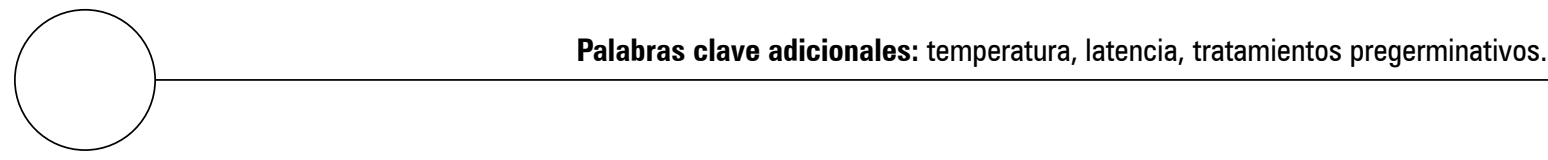

Facultad de Ciencias, Departamento de Biología, Universidad Nacional de Colombia, Bogotá (Colombia).

2 Facultad de Agronomía, Departamento de Agronomía, Universidad Nacional de Colombia, Bogotá (Colombia).

3 Facultad de Agronomía, Programa de Maestría en Ciencias Agrarias, Línea Fisiología de Cultivos, Universidad Nacional de Colombia, Bogotá (Colombia).

4 Autor para correspondencia.nemorenobu@unal.edu.co 


\section{ABSTRACT}

This research evaluated the effect of stratification on the germination of sugar apple seeds from Apulo, Cundinamarca. The effects of wet, cold stratification $\left(8^{\circ} \mathrm{C}\right)(\mathrm{WCS})$ and wet, cold stratification followed by wet, warm stratification $\left(25^{\circ} \mathrm{C}\right.$ ) (WCS +WWS) were evaluated for 15,30 and 45 days for each treatment. After the application of these treatments, germination was tested in growth chambers at 30 and $35^{\circ} \mathrm{C}$ to determine the temperature effect on germination. The seeds had a higher germination rate (GR) and mean velocity of germination (MVG) with the application of WCS for 45 days at $35^{\circ} \mathrm{C}$ with a GR of $65.6 \%$. The seeds kept at $30^{\circ} \mathrm{C}$ germinated later and showed a lower GR and MVG than germination at $35^{\circ} \mathrm{C}$. Based on these results, it can be concluded that the temperature of $35^{\circ} \mathrm{C}$ improved the germination of sugar apple seeds. The response of sugar apple seeds to stratification (WCS and WCS+WWS) could indicate the presence of morpho-physiological dormancy.

Additional key words: temperature, dormancy, pregerminative treatments.

La familia Annonaceae comprende aproximadamente 140 géneros y 2.500 especies con distribución pantropical por debajo de los $2.000 \mathrm{msnm}$. Tres géneros producen frutos comestibles, pero los géneros cultivables más importantes son $A n$ nona y Rollinia (Sanjinés et al., 2006). El género Annona agrupa 150 especies, aunque las de mayor importancia económica son A. squamosa L., A. muricata L., A. cherimola Mill. y el híbrido atemoya (A. squamosa $\times$ A. cherimola); estos frutos son fuente de vitamina $A$, hierro y calorías (Guerrero y Fischer, 2007).

No se conoce con exactitud el origen del anón (A. squamosa L.). Inicialmente fue situado en la India, pero los datos históricos y filogenéticos lo ubican en las regiones tropicales de Centroamérica o las Antillas. Annona squamosa es la especie del género Annona más ampliamente distribuida en el mundo, en Colombia se encuentra en las zonas secas de los valles interandinos en los departamentos de Valle, Caldas, Tolima, Cundinamarca y los Santanderes (Guerrero, 2012).
El anón crece desde el nivel del mar hasta los 1.000 msnm (Hoyos, 1989). El cultivo prospera bien a una temperatura mínima media de $15^{\circ} \mathrm{C}$ y una máxima entre 22 y $28^{\circ} \mathrm{C}$ (Guerrero y Fischer, 2007). Soporta además periodos prolongados de sequía y es probablemente la anonácea más tolerante a esta. Para su desarrollo requiere de una precipitación anual entre 750 y $1.200 \mathrm{~mm}$ (Guerrero, 2012). El cultivo del anón en Colombia es poco tecnificado, los cultivos extensivos establecidos son escasos y el sistema de propagación predominante es vía sexual. La germinación de las semillas de anón tarda entre 1 y 3 meses y sus porcentajes de germinación normalmente son bajos (Hayat, 1963).

El proceso de germinación de semillas presenta tres etapas, durante las cuales el metabolismo celular se incrementa y el embrión reanuda su crecimiento activo, forzando el rompimiento de la cubierta seminal permitiendo la emergencia de la plántula (Bewley, 1997). En la etapa inicial se produce el proceso de imbibición, donde se fa- 
cilita la entrada del agua a la semilla, se activan las enzimas presentes y el sistema de síntesis de proteínas, lo que permite la formación de sustancias necesarias para el desarrollo de la semilla (Chen et al., 2011).

Durante la segunda etapa, la absorción de agua y la respiración son procesos constantes (Bewley y Black, 1994), simultáneamente se hidrolizan el almidón, los lípidos y las proteínas del endospermo en azúcares, ácidos grasos y aminoácidos, que son compuestos simples solubles y movilizables. Posteriormente, estas sustancias se movilizan hacia los puntos de crecimiento del eje embrionario, donde son usadas en los procesos de crecimiento (Sierra, 2005). En la etapa final de la germinación, se produce el crecimiento del embrión por expansión celular y división mitótica, hasta la aparición de la radícula y la plúmula (FinchSavage y Leubner-Metzger, 2006).

La no germinación de las semillas se debe básicamente a dos razones: la quiescencia, que obedece a factores externos no favorables (por baja humedad o temperaturas no adecuadas) para la germinación o por la latencia, que es inherente a factores intrínsecos de la semilla e independiente de las condiciones del medio (Baskin y Baskin, 2004).

Los tipos de latencia existentes son: exógena o de cubierta, se produce porque la testa puede ser muy dura, o impermeable, o por la presencia de sustancias inhibidoras de la germinación (Finch-Savage y Leubner-Metzger, 2006). La morfológica, que se relaciona con la presencia de embriones inmaduros, o rudimentarios poco desarrollados por lo que requieren tiempo para madurar antes de la germinación (Baskin y Baskin, 2001). Otro tipo de latencia es la interna que es controlada en el interior de los tejidos por dos fenómenos independientes: la semipermeabilidad de la cubierta de la semilla y la presencia de un mecanismo fisiológico inhibidor (Finch-Savage y Leubner-Metzger, 2006). Igual- mente se puede encontrar la latencia morfofisiológica, que se presenta cuando el embrión no está desarrollado en su totalidad y además existen mecanismos fisiológicos fuertes que inhiben la germinación (Baskin y Baskin, 2004). En la familia Annonaceae existen indicios de que las semillas presentan latencia morfológica y fisiológica (Baskin y Baskin, 2001), y específicamente en A. squamosa se ha reportado latencia morfológica (diversos autores, citados por Baskin y Baskin. 2001).

Algunos tratamientos que se efectúan antes de la germinación y que permiten superar la latencia, son denominados pregerminativos. La estratificación, permite superar la latencia relacionada con la presencia de un embrión inmaduro que es incapaz de germinar con normalidad (Baskin y Baskin, 2001), debido a que promueve el incremento transitorio de la concentración de giberelinas y citoquininas en las semillas (Solís, 2006). El aumento de las giberelinas endógenas, como resultado de la estratificación, estimula la germinación de semillas y además se ha reportado que favorece la reducción de ácido abscísico (ABA) (Bewley y Black, 1994). La estratificación en altas temperaturas (entre 22 y $30^{\circ} \mathrm{C}$ ) o a bajas temperaturas (entre 0 y $8^{\circ} \mathrm{C}$ ) consiste en colocar las semillas en un sustrato húmedo durante un periodo de tiempo que sea adecuado para la especie (Baskin et al., 2002). Generalmente la estratificación en frío consiste en disponer las semillas junto a un medio que conserve la humedad, arena o turba, y almacenarlas en un recipiente plástico con tapa al frío, durante un periodo de tiempo que varía de unas pocas semanas a unos meses.

La temperatura es considerada uno de los factores más importantes en la germinación de la mayoría de las especies vegetales (Probert, 2000). Los resultados sugieren que la estratificación en frío actúa como un promotor de la germinación y se puede deber a adaptaciones al ambiente natural, en los cuales, se originó la especie (Manjkhola et al., 2003). 
En Brasil se han realizado estudios con el fin de establecer que métodos pregerminativos son útiles para potenciar la germinación de A. squamosa; Ferreira et al. (2002) reportaron que la aplicación de giberelinas en una concentración de $250 \mathrm{mg}$ $\mathrm{L}^{-1}$, permitió porcentajes de germinación de las semillas del 77\%; Stenzel et al. (2003) alcanzaron porcentajes de germinación en $A$. squamosa del 75\% mediante escarificación mecánica con lija en el extremo basal de las semillas, seguido por la aplicación de $50 \mathrm{mg} \mathrm{L}^{-1}$ de giberelinas.

En anón existe poca información sobre tratamientos pregerminativos que mejoren la germinación a través del rompimiento de latencia, en esta investigación se evaluó el efecto de la estratificación como tratamiento pregerminativo sobre la germinación de semillas de A. squamosa.

\section{MATERIALES Y MÉTODOS}

La investigación se realizó en el laboratorio de Fisiología de Cultivos de la Facultad de Agronomía, Universidad Nacional de Colombia, Bogotá. Se emplearon semillas de anón (Annona squamosa L.), obtenidas de frutos cosechados de las accesiones denominadas C2AS224, C2AS225 y C2AS226 procedentes de cultivos ubicados en la región de Apulo, Cundinamarca, a 674 msnm. Los frutos maduros cosechados, se lavaron con agua a temperatura ambiente. Se retiró manualmente la semilla de la pulpa, posteriormente lavadas y colocadas en toallas de papel absorbente para secarlas a temperatura ambiente (media: 18,7; máxima: 22,9; mínima: 17,3) durante 72 h.

La siembra de las semillas a tratar se hizo sobre turba rubia Klassman ${ }^{\circledR}$ sin nutrientes, caracterizada por su alta retención de agua y su capacidad de aireación. Con el fin de evitar la presencia de patógenos, tanto la turba como las semillas fueron desinfectadas. La desinfección de la turba se realizó con Benomyl $\left(1 \mathrm{~g} \mathrm{~L}^{-1}\right)$ y Vitavax $\left(2 \mathrm{~g} \mathrm{~L}^{-1}\right)$. Las semillas se desinfectaron por inmersión en hipoclorito de sodio al 1\% durante 9 min, lavadas con agua destilada y etanol al 96\%, finalmente fueron lavadas con agua destilada para retirar el etanol que pudiera quedar en la semilla.

Para evaluar el efecto de la estratificación sobre la germinación se realizó un ensayo en un diseño completamente aleatorizado en un arreglo factorial de $2 \times 3 \times 2$ donde los factores son dos tipos de estratificación (frío y frío+caliente), tres tiempos $(15,30$ y $45 \mathrm{~d})$ y dos temperaturas $(30$ y $35^{\circ} \mathrm{C}$ ) en dos fitotrones (Conviron CMP3244, Winnipeg, Canadá), en oscuridad total, con cinco repeticiones y 25 semillas por tratamiento. En el control no se realizó ningún tipo de estratificación, cinco repeticiones de 25 semillas fueron llevadas a dos fitotrones Conviron CMP3244 con dos temperaturas $\left(30\right.$ y $\left.35^{\circ} \mathrm{C}\right)$ (tabla 1 ).

Se realizaron observaciones cada $5 \mathrm{~d}$ durante 30 $\mathrm{d}$, ya que las semillas germinadas después de este periodo pueden considerarse latentes (Baskin y Baskin, 2001). Se registraron como semillas germinadas aquellas en las que hubo protrusión radicular y alargamiento de la radícula mayor de $3 \mathrm{~mm}$.

\begin{tabular}{|c|c|c|}
\hline \multicolumn{3}{|c|}{$\begin{array}{l}\text { Tabla 1. Diseño de tratamientos de estratificación } \\
\text { húmeda fría (EHF) y frío más caliente } \\
\text { (EHF+EHC) a diferentes tiempos, para } \\
\text { semillas de anón. }\end{array}$} \\
\hline Tratamiento & Tiempo (días) & Temperatura $\left({ }^{\circ} \mathrm{C}\right)$ \\
\hline \multirow{2}{*}{ Control } & & 30 \\
\hline & & 35 \\
\hline \multirow{6}{*}{ EHF } & 15 & 30 \\
\hline & & 35 \\
\hline & 30 & 30 \\
\hline & & 35 \\
\hline & 45 & 30 \\
\hline & & 35 \\
\hline \multirow{6}{*}{$E H F+E H C$} & 15 & 30 \\
\hline & & 35 \\
\hline & 30 & 30 \\
\hline & & 35 \\
\hline & 45 & 30 \\
\hline & & 35 \\
\hline
\end{tabular}


Las semillas no germinadas se clasificaron como viables y no viables mediante la metodología de tinción con tetrazolio (ISTA, 1996), esta evaluación se realizó mediante el corte longitudinal ventral de las semillas no germinadas, sumergidas en tetrazolio al $1 \%$ durante un periodo mayor a 24 h en completa oscuridad a $32^{\circ} \mathrm{C}$ y $60 \%$ de humedad.

Luego de las evaluaciones se calculó el porcentaje de germinación (PG), la velocidad media de germinación (VMG), el tiempo medio de germinación (TMG) y la frecuencia de germinación (tabla 2). Las variables respuesta PG y VMG, fueron transformadas con raíz cuadrada, debido a que los datos no se distribuían normalmente según prueba Shapiro-Wilks $(P \leq 0,05)$.

Se realizó un Anova y una prueba de comparación de medias múltiples de Tukey, con un 95\% de confianza, para cada factor que presentó diferencias en la prueba de Anova. Para el procesamiento y análisis de los datos se usaron los paquetes estadísticos Statistica v.10 y Origin Pro 8.5.1 SR2 (Origin Lab Corporation).

\section{RESULTADOS}

Los tratamientos pregerminativos de estratificación incidieron significativamente tanto en el porcentaje de germinación (PG) como en la velocidad media de germinación (VMG). Comparados con el testigo, los tratamientos EHF 45 y $\mathrm{EHF}+\mathrm{EHC} 30+30$ a $35^{\circ} \mathrm{C}$, incrementaron el PG de $23,2 \%$ a $65,6 \%$ y $45,6 \%$ respectivamente. Por otro lado, las semillas sometidas a tratamientos de $30^{\circ} \mathrm{C}$ mostraron PG más bajos que la mayoría de aquellas con tratamientos a $35^{\circ} \mathrm{C}$, sin presentar diferencias significativas con el testigo (figura 1).

Las mayores velocidades de germinación se alcanzaron con los tratamientos EHF 45 y EHF+EHC $30+30$ con temperaturas de $35^{\circ} \mathrm{C}$, con valores de 1,28 y 1,32 respectivamente, comparados con el testigo que tuvo una VMG de 0,56. Los tratamientos a temperaturas de $30^{\circ} \mathrm{C}$ no mostraron diferencias significativas con el testigo (figura 2).

El análisis de varianza realizado para la variable tiempo medio de germinación (TMG), indicó que la temperatura incide directamente en el tiempo necesario para que germinen las semillas. Cuando la temperatura utilizada fue de $35^{\circ} \mathrm{C}$ se reduce significativamente el tiempo de germinación de las semillas respecto a los tratamientos a $30^{\circ}$ (figura 3). Las frecuencias de germinación (figura 4) de los tratamientos desarrollados a $30^{\circ} \mathrm{C}$ muestran que a diferencia del testigo, todos los tratamientos incrementan su germinación cerca o sobre los 30 $\mathrm{d}$, lo que indica que la germinación posiblemente continúa más allá del tiempo de evaluación. Las pruebas de viabilidad realizadas al finalizar este ensayo tomaron valores entre $60 \%$ y $88 \%$.

\section{DISCUSIÓN}

Dentro de los factores ambientales que afectan la germinación de las semillas, la temperatu-

Tabla 2. Fórmulas utilizadas para el cálculo de variables de estudio.

\begin{tabular}{|l|l|}
\hline \multicolumn{1}{|c|}{ Variable } & \multicolumn{1}{c|}{ Fórmula } \\
\hline Porcentaje de germinación $(\mathrm{PG})$ & $\mathrm{PG}=$ No. de semillas germinadas/ No. de semillas incubadas \\
\hline Velocidad media de germinación $(\mathrm{VMG})$ & $(\mathrm{VMG})=\mathrm{P} 1 / \mathrm{T} 1+\mathrm{P} 2 / \mathrm{T} 2+\ldots . .+\mathrm{Pn} / \mathrm{Tn}$ \\
\hline Tiempo medio de germinación (TMG) & $(\mathrm{TMG})=((\mathrm{x} 1 \mathrm{~d} 1)+(\mathrm{x} 2 \mathrm{~d} 2)+\ldots(\mathrm{xn} \mathrm{dn})) / \mathrm{Xn}$ \\
\hline Frecuencia de germinación & Frecuencia $=\mathrm{Xi} / \mathrm{Xn}$ \\
\hline
\end{tabular}

Donde: $\mathrm{P}=$ número de semillas germinadas; $\mathrm{T}=$ tiempo germinación; $\mathrm{n}=$ día último control; $x 1, x 2, x 15=$ semillas germinadas día $1,2, \ldots n ; \mathrm{d1}, \mathrm{d2}, \ldots$ $\mathrm{dn}=$ días incubación, $\mathrm{Xi}=$ semillas germinadas por día de revisión y $\mathrm{Xn}=$ número total de semillas germinadas el último día de control. Fuente: Ranal y García De Santana, 2006. 


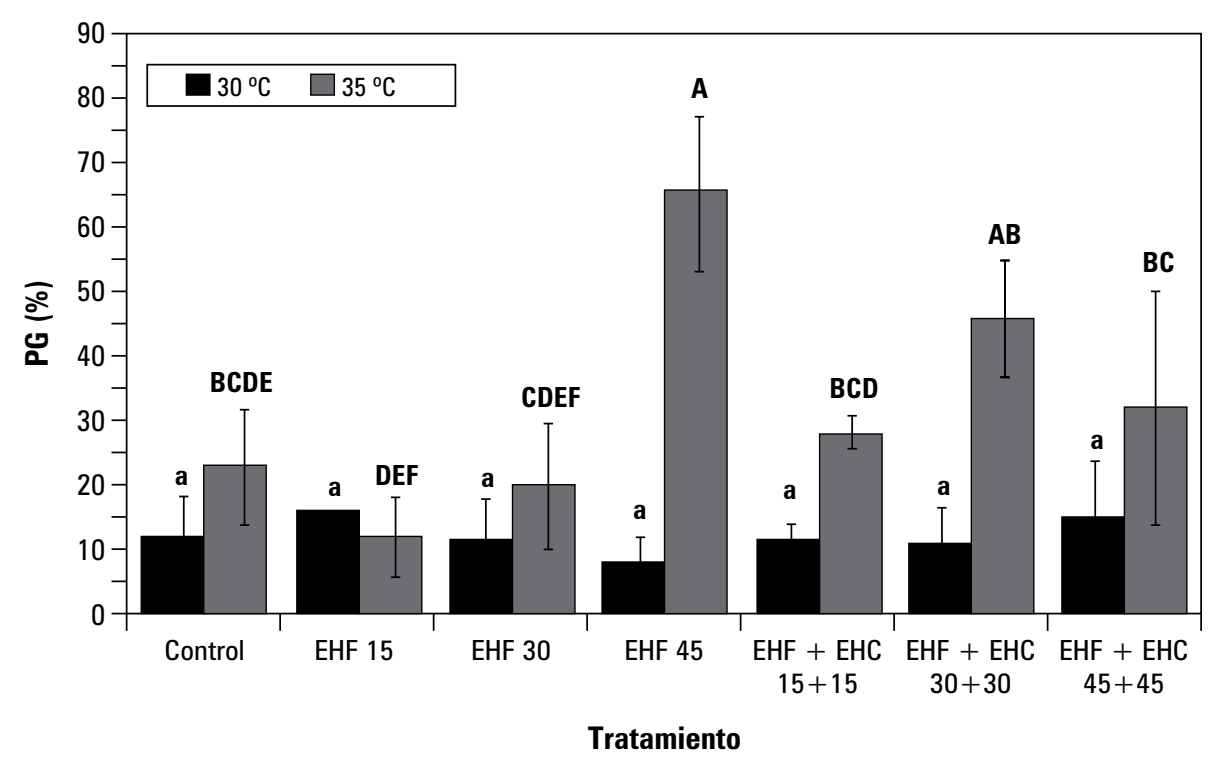

Figura 1. Efecto de la estratificación húmeda fría (EHF) y húmeda fría + húmeda caliente (EHF+EHC) sobre el porcentaje de germinación (PG) de semillas de anón. Promedios con letras distintas indican diferencia significativa según la prueba de Tukey $(P \leq 0,05)$; mayúsculas para los tratamientos a $35^{\circ} \mathrm{C}$ y minúsculas para los tratamientos a $30^{\circ} \mathrm{C}$. Las barras sobre las columnas indican desviación estándar.

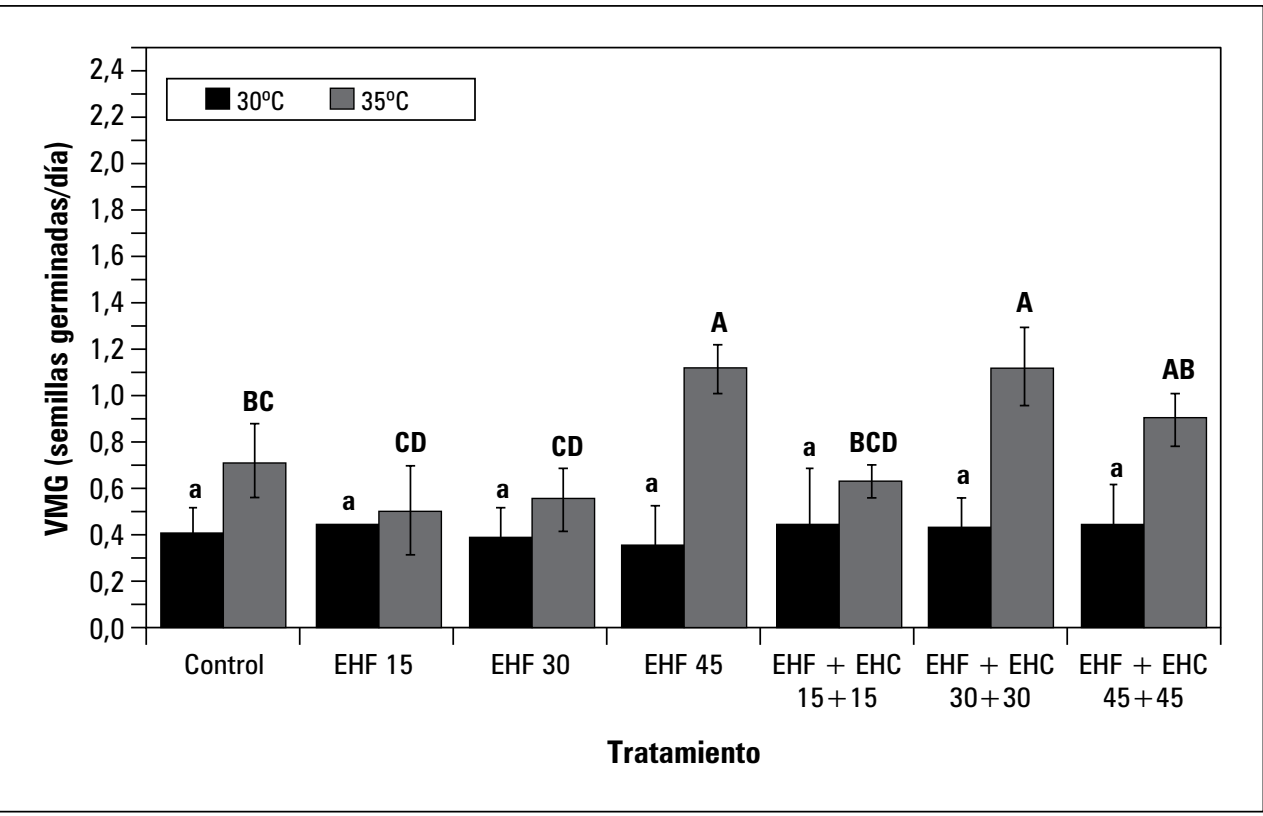

Figura 2. Efecto de la estratificación húmeda fría (EHF) y húmeda fría + húmeda caliente (EHF+EHC) sobre la velocidad media de germinación (VMG) de semillas de anón. Promedios con letras distintas indican diferencia significativa según prueba de Tukey $(P \leq 0,05)$; mayúsculas para los tratamientos a $35^{\circ} \mathrm{C}$ y minúsculas para los tratamientos a $30^{\circ} \mathrm{C}$. Las barras sobre las columnas indican desviación estándar. 
ra es quizá el factor más importante (Baskin y Baskin, 2003), y su efecto puede ser evaluado a partir de los cambios que ocasiona sobre el porcentaje, la velocidad y el tiempo de germinación (Alves et al., 2006).

En este estudio se encontró que la temperatura afectó de forma positiva la germinación de las semillas, especialmente aquellas establecidas a temperatura de $35^{\circ} \mathrm{C}$, donde sin importar el tratamiento al que fueron sometidas, se incrementó el porcentaje y la velocidad de la germinación, a la vez que se redujo el tiempo de la misma; por otro lado, las semillas establecidas a $30^{\circ} \mathrm{C}$ tardaron al menos $16 \mathrm{~d}$ en germinar y solo alcanzaron porcentajes de germinación inferiores al 20\%. Autores como Bewley y Black (1994) concluyen que la germinación de las semillas solo se presenta en límites bien definidos de temperatura, y que estos límites son característicos de cada especie, por lo que se requiere determinar las temperaturas mínima, óptima y máxima, siendo la temperatura óptima aquella donde se alcanza el máximo porcentaje de germinación en menor tiempo (Vilar et al., 2005). En semillas con buen vigor las temperaturas máximas incrementan la velocidad de germinación (Nassif y Perez, 2000). Por otro lado, las temperaturas mínimas reducen la velocidad de germinación y afectan los porcentajes de germinación (Carvalho y Nakagawa, 2000). A pesar de que los resultados obtenidos indican que la temperatura de $35^{\circ} \mathrm{C}$ es más adecuada para la germinación de las semillas de anón comparada con $30^{\circ} \mathrm{C}$, en la literatura se reportan porcentajes de germinación superiores al $75 \%$ a $25^{\circ} \mathrm{C}$ e incluso a temperaturas alternas de $20-30^{\circ} \mathrm{C}$ (Ferreira et al., 2002; Stenzel et al., 2003). Considerando las respuestas observadas a $35^{\circ} \mathrm{C}$ y la no reducción del porcentaje de germinación, no se puede inferir que esta sea la temperatura máxima en la que el anón puede germinar (Nassif y Pérez, 2000). Estos resultados corroboran que el proceso germinativo, es un aspecto dependiente de la especie, del genotipo dentro

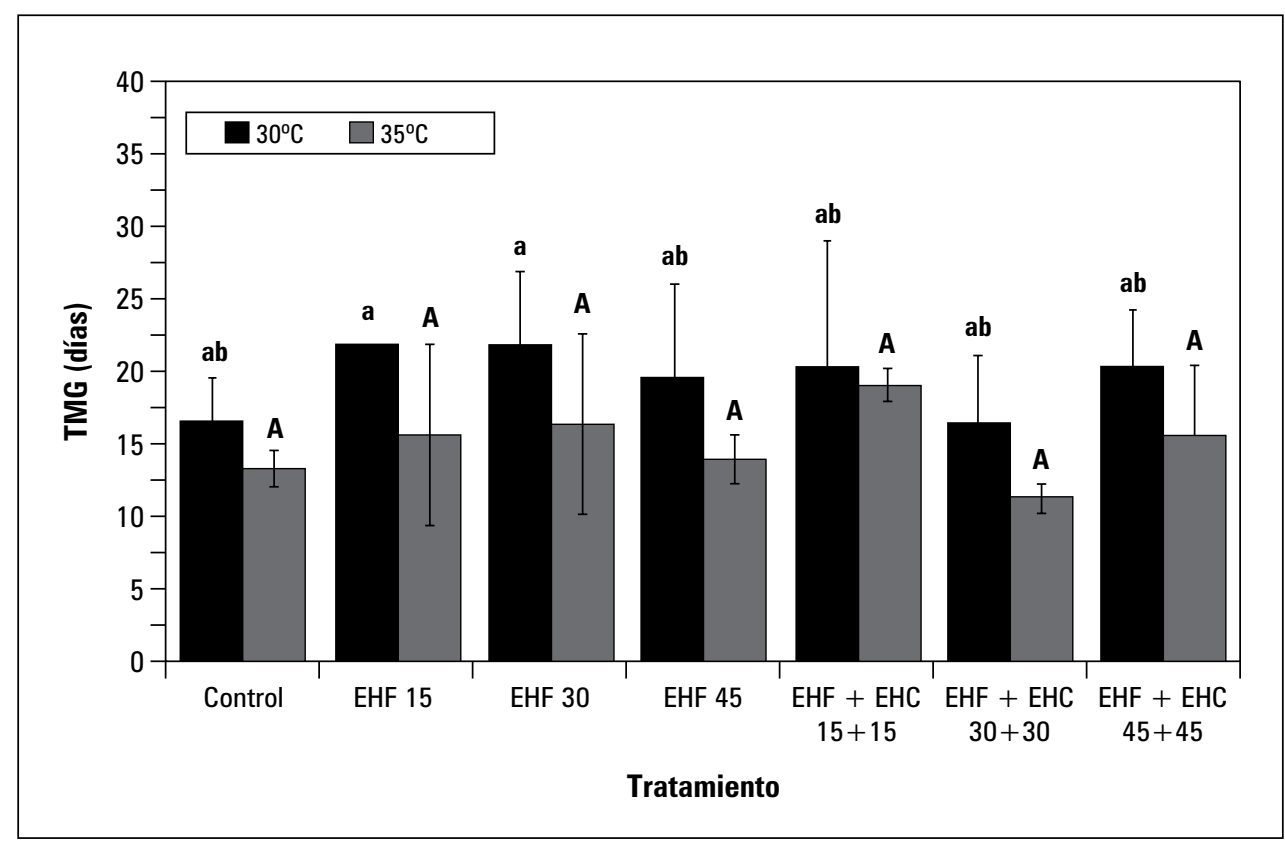

Figura 3. Efecto de la estratificación húmeda fría (EHF) y húmeda fría + húmeda caliente (EHF+EHC) sobre la tiempo medio de germinación (TMG) de anón. Promedios con letras distintas indican diferencia significativa según prueba de Tukey $(P \leq 0,05)$; mayúsculas para los tratamientos a $35^{\circ} \mathrm{C}$ y minúsculas para los tratamientos a $30^{\circ} \mathrm{C}$. Las barras sobre las columnas indican desviación estándar. 
de cada taxón y del conjunto de condiciones en las cuales se incuba la semilla (Lobo et al., 2007). Dado que existe una gran variabilidad genética dentro de esta especie (Guerrero y Fischer, 2007), y que las semillas de $A$. squamosa obtenidas en la población de Apulo, corresponden genomas diferentes a los utilizados en Brasil.
Las semillas establecidas a $30^{\circ} \mathrm{C}$ incrementan su porcentaje de germinación hacia el final del periodo de evaluación, lo que indica que a esta temperatura las semillas podrían alcanzar mayores valores de germinación y que se requiere un mayor tiempo de evaluación. Estudios realizados en otras anonáceas como chirimoya y
A

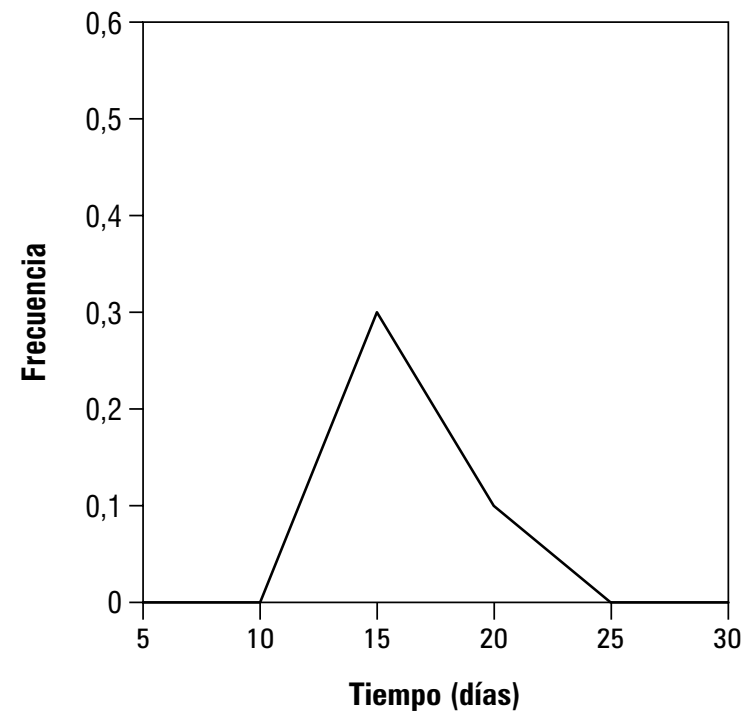

C

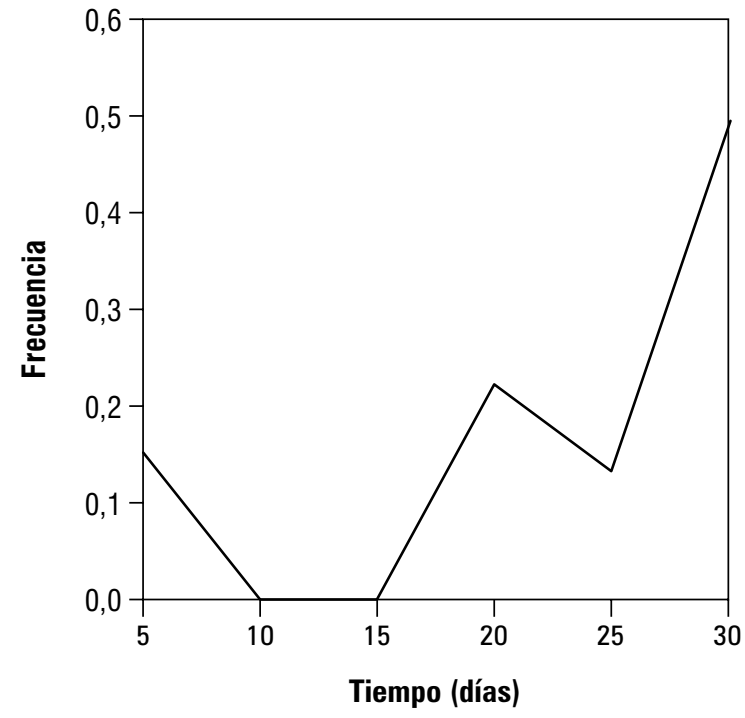

B

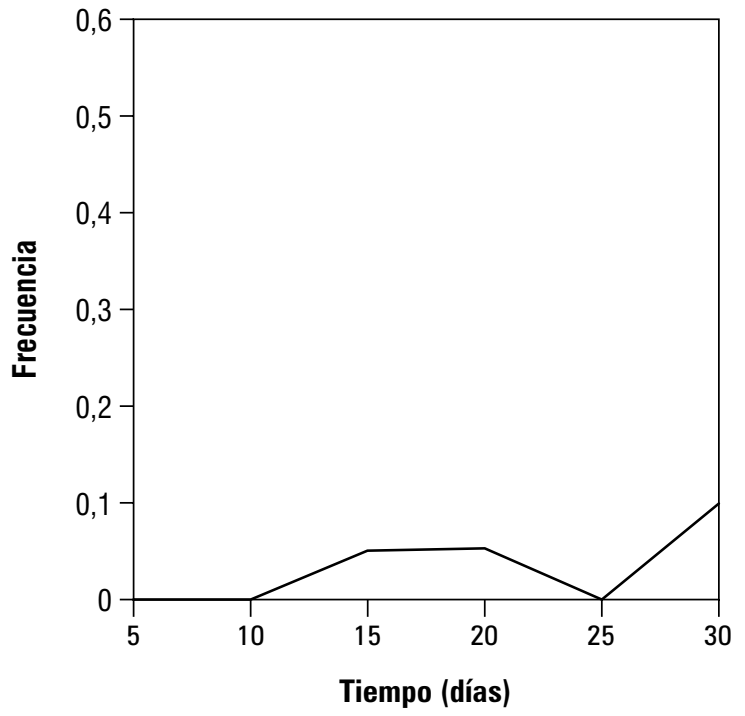

D

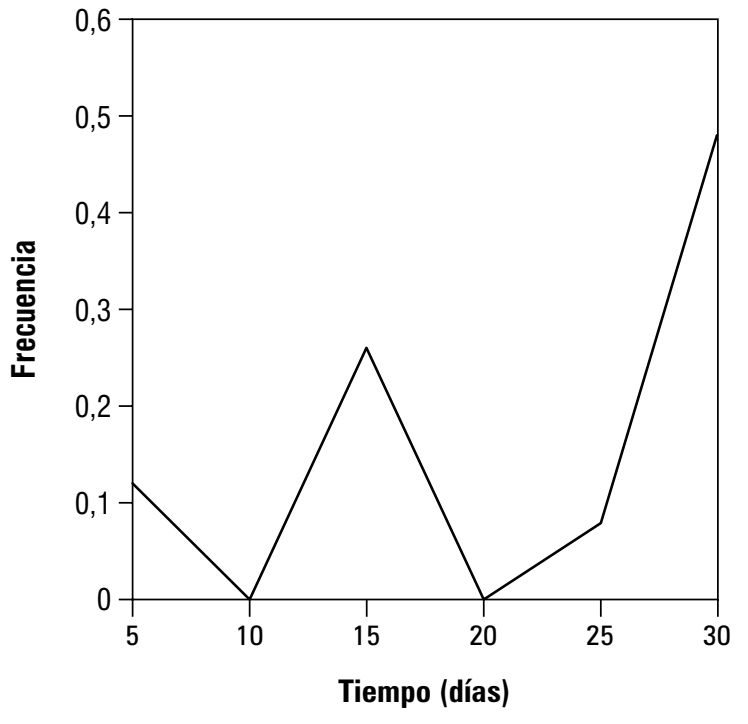

Figura 4. Efecto de los tratamientos de estratificación (EHF y EHF+EHC) sobre las frecuencias de germinación de semillas de anón establecidas a $30^{\circ} \mathrm{C}$. A. Control, B. EHF 15. C. EHF 30, D. EHF 45, E. EHF + EHC 15+15, F. EHF + EHC 30+30 y G. EHF + EHC 45+45. (Continúa en la siguiente página) 


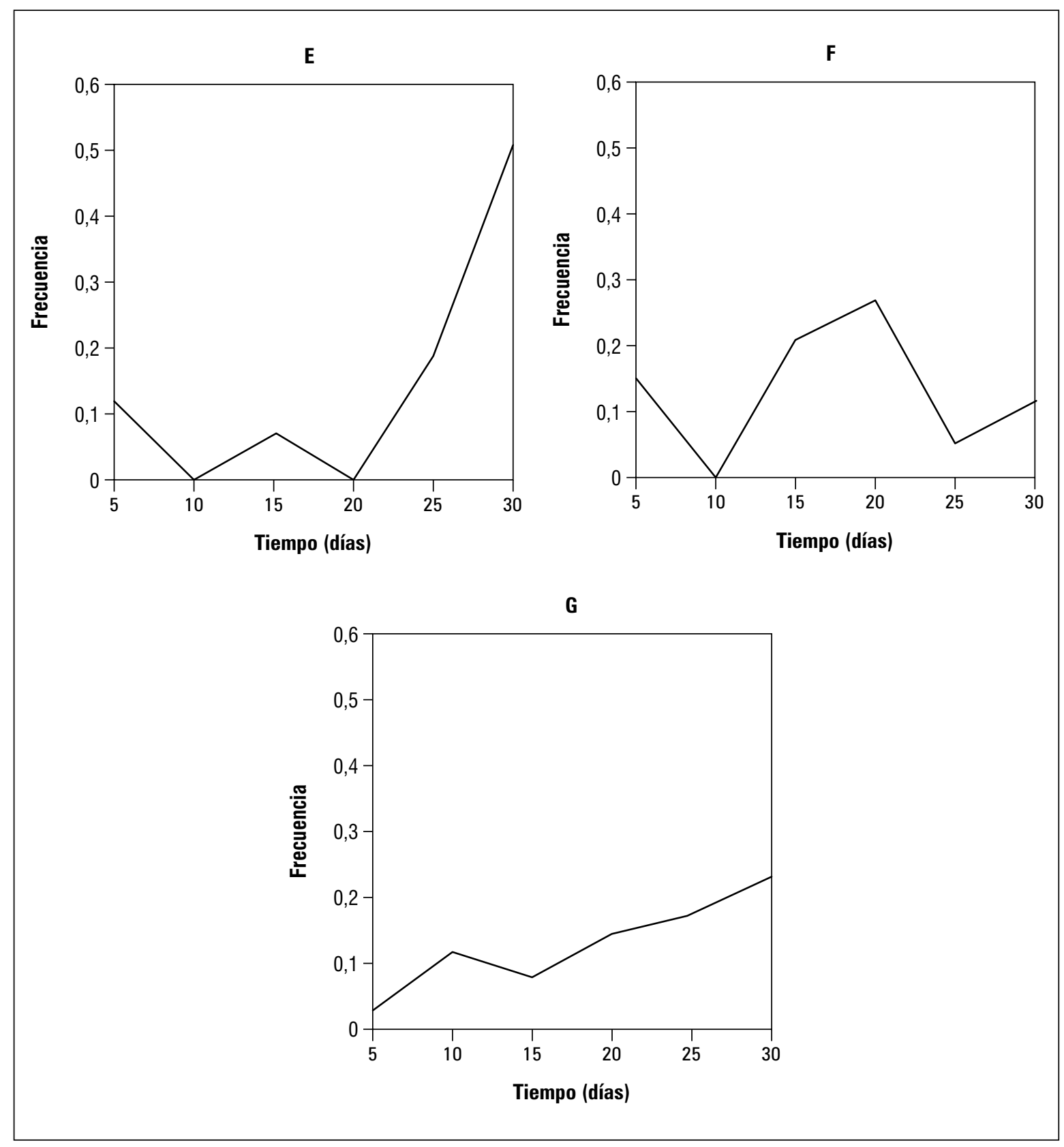

Figura 4. Efecto de los tratamientos de estratificación (EHF y EHF+EHC) sobre las frecuencias de germinación de semillas de anón establecidas a $30^{\circ}$ C. A. Control, B. EHF 15. C. EHF 30, D. EHF 45, E. EHF + EHC 15+15, F. EHF + EHC $30+30$ y G. EHF + EHC 45+45.

guanábana, indican que la latencia puede superarse con la aplicación simultánea de estratificación húmeda caliente en un periodo mayor a 90 d y adición exógena de giberelinas (Lobo et al., 2007). Contrario a lo reportado por Baskin y Baskin (2001), el efecto positivo de los tratamientos de estratificación EHF 45 y EHF+EHC
30+30 en esta investigación podría indicar la presencia de una latencia morfofisiológica y no únicamente morfológica. La estratificación causa cambios metabólicos y fisiológicos en la semilla que involucran tanto al embrión como a las capas que lo recubren (Leubner-Metzger, 2005). Chien et al. (1998) reportaron que la es- 
tratificación cálida induce la maduración de los embriones poco desarrollados lo que permite superar la latencia morfológica. Mientras tanto, la estratificación fría promueve el crecimiento del embrión, reduce la sensibilidad y el contenido de ácido abscísico y simultáneamente incrementa la concentración y sensibilidad de las semillas al ácido giberélico $\left(\mathrm{AG}_{3}\right)$ lo que supera la latencia fisiológica (Bewley y Black, 1994).
Finalmente, se concluye que la temperatura de germinación de $35^{\circ} \mathrm{C}$ incrementó el porcentaje y velocidad de la germinación y redujo el tiempo de la misma. Por otro lado, la estratificación húmeda fría durante 45 d y la estratificación húmeda fría por 30 d seguida por 30 d de estratificación húmeda cálida, permitió obtener mayores porcentajes y velocidades de germinación.

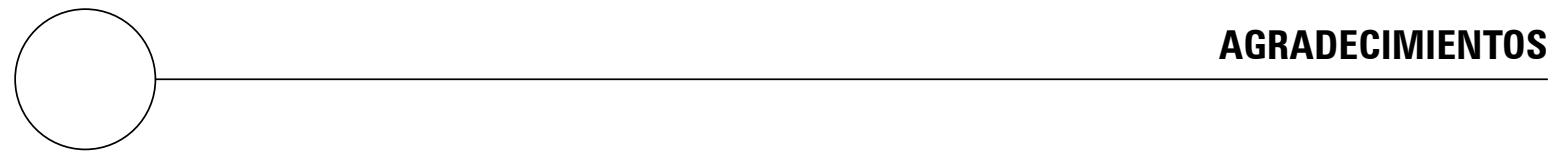

Este proyecto se realizó gracias al apoyo financiero de la Dirección de Investigación (DIB) de la Universidad Nacional de Colombia, Bogotá, y al programa Material de Siembra y Mejoramiento Genético del Anón (Annona squamosa L.), proyecto "Establecimiento del Banco Colombiano de Germoplasma Colombiano de Anón (Annona squamosa L.)", financiado con recursos del Ministerio de Agricultura y Desarrollo Rural, Bogotá.

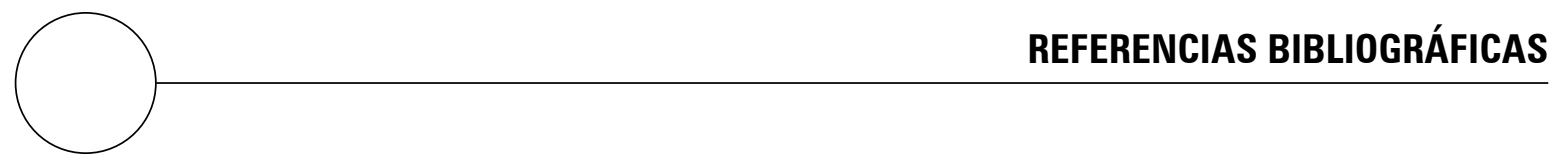

Alves, G., V. Pontes, A. Alves, A. Da Silva y A. Medeiros. 2006. Viabilidade de sementes de acerola ( $\mathrm{Mal}$ pighia punicifolia DC) influenciada pelo substrato, temperatura e coloração de frutos. Pesquisa Agropecuária Tropical 36(1), 7-11.

Baskin, C.C. y J.M. Baskin. 2001. Seeds. Ecology, biogeography, and evolution of dormancy and germination. Academic Press, San Diego, CA.

Baskin, C.C. y J.M. Baskin. 2003. When breaking seed dormancy is a problem try a move-along experiment. Native Plants J. 4(1), 17-21.

Baskin, C.C., O. Zackrisson y J.M. Baskin. 2002. Role of warm stratification in promoting germination of seeds of Empetrum hermaphroditum (Empetraceae), a circumboreal species with a stony endocarp. Amer. J. Bot. 89(3), 486-493.

Baskin, J.M. y C.C. Baskin. 2004. A classification system for seed dormancy. Seed Sci. Res. 14, 1-16.
Bewley, J.D. y M. Black. 1994. Seeds: physiology of development and germination. Plenum Press, New York, NY.

Bewley, J.D. 1997. Seed germination and dormancy. Plant Cell 9, 1055-1066.

Carvalho, N.M. y J. Nakagawa. 2000. Sementes: ciência tecnologia e produção. 4a ed. FUNEP, Jaboticabal, Brasil.

Chien, C., L. Kuo-Huang y T.P. Lin. 1998. Changes in ultrastructure and abscisic acid level, and response to applied gibberellins in Taxus mairei seeds treated with warm and cold stratification. Ann. Bot. 81, 41-47.

Chen, F., R. Martin, S. Song y H. Nonogaki. 2011. Seed development and germination. Plant Tissue Cult. Dev. Biotecnol. 9, 127-140.

Ferreira, G., P.R. Erig y E. Moro E. 2002. Uso de ácido giberélico em sementes de fruta-do-conde (Annona 
squamosa L.) visando à produção de mudas em diferentes embalagens. Rev. Bras. Frutic. 24(1), 178-182.

Finch-Savage, W.E. y G. Leubner-Metzger. 2006. Seed dormancy and the control of germination. New Phytologist 171, 501-523.

Guerrero, E.J. 2012. Anón (Annona squamosa L.). pp. 915-927. En: Fischer, G. (ed.). Manual para el cultivo de frutales en el trópico. Produmedios, Bogotá.

Guerrero, E. y G. Fischer. 2007. Manejo integrado en el cultivo de anón (Annona squamosa L.). Rev. Colomb. Cienc. Hortic. 2(1), 154-169.

Hayat, M.A. 1963. Morphology of seed germination and seedling in Annona squamosa. Bot. Gazette 124(5), 360-362.

Hoyos, J. 1989. Frutales en Venezuela. Sociedad de Ciencias Naturales La Salle, Caracas. pp. 35-48.

ISTA. 1996. International rules for seed testing, 1996. International Seed Testing Association. Seed Sci. Technol. 24 (Supplement). Zurich, Suiza.

Leubner-Metzger. 2005. Beta-1,3-Glucanase gene expression low-hydrated seeds as a mechanism for dormancy release during tobacco after-ripening. Plant J. 41, 133-145.

Lobo, M., O. Delgado, J.R. Cartagena, E. Fernández y C.I. Medina. 2007. Categorización de la germinación y la latencia en semillas de chirimoya (Annona cherimola L.) y guanábana (Annona muricata L.), como apoyo a programas de conservación de germoplasma. Agron. Colomb. 25(2), 231-244.

Manjkhola, S., U. Dhar y R.S. Rawal. 2003. Treatments to improve seed germination of Arnebia benthamii: an endangered medicinal herb of high altitude $\mathrm{Hi}$ malaya. Seed Sci. Technol. 31, 571-577.

Nassif, S. y S. Perez. 2000. Efeitos da temperatura na germinação de sementes de Amendoim-DoCampo (Pterogyne nitens Tul.). Rev. Bras. Sementes $22(1)$, p.1-6.

Probert, R.J. 2000. The role of temperature in the regulation of seed dormancy and germination. In: Fenner, M. (ed.), Seeds: The ecology of regeneration in plant communities. $2^{\text {nd }}$ ed. Cabi Publishing, Wallingford, UK.

Ranal, M.A. y D. Garcia De Santana. 2006. How and why to measure the germination process?. Rev. Bras. Bot. 29(1), 1-11.

Sanjinés, A., B. Øllgaard y H. Balslev. 2006. Frutos comestibles. Botánica económica de los Andes centrales. Universidad Mayor de San Andrés, La Paz. pp. 329-346.

Sierra P., J. 2005. Fundamentos para el establecimiento de pasturas y cultivos forrajeros. Universidad de Antioquia, Medellín, Colombia.

Solís F., A.A. 2006. Algunos factores que influyen sobre la germinación de semillas de borraja (Borago officinalis L.). Universidad de Concepción, Chillán, Chile.

Stenzel, N.M.C., Murata, I.M. y C.S.V.J. Nemes. 2003. Superação da dormência em sementes de atemóia e fruta-do-conde. Rev. Bras. Frutic. 25(2), 305-308.

Vilar, I., R.A. De Andrade y A. Baldo. 2005. Influência da temperatura na germinação de sementes de Annona montana. Rev. Bras. Frutic. 27(2), 344-345. 